\title{
Produção de etanol a partir de lactossoro industrial
}

\author{
Isanna M. Florêncio', Eliane R. Florentino', Flávio L. H. da Silva², \\ Ricardo S. Martins ${ }^{3}$, Mônica T. Cavalcanti ${ }^{4}$ \& Josivanda P. Gomes ${ }^{5}$
}

\begin{abstract}
RESUMO
Entre os principais subprodutos do setor de laticínios se encontra o soro de queijo, obtido após precipitação da caseína do leite. Com o avanço da tecnologia a elaboração de queijos passou de um processo tradicional para um processo industrial no qual são produzidos diariamente milhares de litros de soro. A fermentação do soro do queijo objetivando a produção de etanol pode apresentar-se como alternativa tecnicamente viável pois, além de reduzir o potencial poluidor deste resíduo, ainda pode gerar um produto de maior valor agregado. Desta forma, o objetivo deste trabalho foi a obtenção do etanol utilizando-se o soro de queijo "tipo coalho", desproteinizado, como substrato para a levedura Saccharomyces cerevisiae. Foram utilizadas a ferramenta de planejamento fatorial e a análise de superfície de resposta para otimização do processo estudando-se diferentes concentrações de levedura e percentagens de sacarose. O melhor resultado foi obtido ao se adicionar $100 \mathrm{~g} \mathrm{~L}^{-1}$ de sacarose e $6,0 \mathrm{~g} \mathrm{~L}^{-1}$ de levedura ao soro obtendo-se uma porcentagem de conversão de substrato em etanol de $76,14 \%$.
\end{abstract}

Palavras-chave: soro de queijo, fermentação alcoólica, etanol

\section{Production of ethanol from industrial whey}

\begin{abstract}
Among the main sub-products of the dairy industry is the cheese whey, obtained after milk's casein precipitation. With the advancement of technology, the production of cheese passed from a traditional process to a manufacturing process where everyday thousands of liters of whey are produced. The fermentation of cheese whey with the purpose of producing ethanol can present itself as a technically feasible alternative, as well as besides reducing the pollution potential of this residue it can still generate a product with higher added value. Thus, the aim of this paper was to obtain ethanol, using the cheese whey "sort of curd cheese", deproteinized, as a substrate for the yeast Saccharomyces cerevisiae and the addition of sucrose. A statistical design was used and different inoculum concentrations and percentages of sucrose were studied. The best result was obtained by adding $100 \mathrm{~g} \mathrm{~L}^{-1}$ of sucrose and $6.0 \mathrm{~g} \mathrm{~L}^{-1}$ of inoculum into the whey getting a \% conversion of substrate to ethanol of $76.14 \%$.
\end{abstract}

Key words: cheese whey, alcoholic fermentation, ethanol

19 DQ/UEPB, Rua Baraúnas, 35, Bairro Universitário, CEP 58429-5000, Campina Grande, PB. Fone: (83) 3315-336. E-mail: isannamenezes@hotmail.com; elianerf@yahoo.com.br

${ }^{2}$ CT/UFPB, Cidade Universitária, João Pessoa, PB. Fone: (83) 3216-7119. E-mail: flavioluizh@yahoo.com.br

${ }^{3}$ NUPPA/UFPB, Cidade Universitária, João Pessoa, PB. Fone: (83) 3216-7200. E-mail: ricardomartins.nuppa@gmail.com

${ }^{4}$ UATA/UFCG, Rua Jairo Vieira Feitosa, 1770, Bairro Pereiros, CEP 58840-000, Pombal, PB. Fone: (83) 3431-4000, Ramal 4026. E-mail: monicatejoc@yahoo.com.br

${ }_{5}^{5}$ UAEA/UFCG, Av. Aprígio Veloso, 882, Bairro Bodocongó, CEP 58429-900, Campina Grande, PB. Fone: (83) 2101-1055. E-mail: josivanda@gmail.com 


\section{INTRODUÇÃO}

O queijo é um dos derivados do leite mais produzidos e consumidos no mundo. Na sua produção ocorre eliminação do lactossoro, usualmente conhecido como soro de queijo, que também pode ser obtido pela extração da caseína (Florentino et al., 2005). O soro constitui a porção ou fase aquosa do leite, resultante da dessora do coágulo e pode ser caracterizado como um líquido amarelo-esverdeado e, dependendo do tipo de queijo, pode possuir sabor ligeiramente ácido ou doce (Abreu, 2005).

O queijo Coalho é fabricado há mais de 1 século fazendose uso da matéria-prima leite de vaca cru ou pasteurizado, em vários Estados da Região Nordeste do Brasil e é tradicionalmente consumido fresco ou maturado (Cavalcante et al., 2007).

Para a produção de $1 \mathrm{~kg}$ de queijo são necessário $10 \mathrm{~L}$ de leite resultando, com isto, em $9 \mathrm{~L}$ de soro, ou seja, cerca de 85 a $95 \%$ do volume de leite utilizado na fabricação de queijos resultam em soro, o qual contém aproximadamente metade dos sólidos do leite, sendo eles: proteínas solúveis, nitrogênio não proteico, sais minerais, vitaminas e, principalmente, a lactose (Santos \& Ferreira, 2001; Richards, 2002).

São constantes os esforços para aproveitamento de resíduos agroindustriais em todo o mundo. Em particular o soro do leite, pela abundância de produção, características nutricionais e elevada capacidade poluente, tem sido, há tempo, motivo de vários estudos (Silva \& Herman-Gomez, 2000; Valduga et al., 2006; Madrona et al., 2009; Gabardo et al., 2011). Contudo, faz-se necessário o estudo de formas para conservá-lo e utilizálo minimizando problemas tanto de ordem ambiental como nutricional, em benefício da fauna, flora, animais domésticos e do próprio homem, utilizando o soro como alimento de alto valor nutricional.

As aplicações do soro são inúmeras, englobando as indústrias de lácteos, carnes, misturas secas (para condimentar), panificação (melhorar o valor nutricional, sabor e cor), chocolate, cereais, aperitivos, bebidas, material combustível, fertilizante e alimentação animal, entre outros. Em virtude do seu alto valor nutricional o soro de queijo se torna um substrato atraente para sua utilização em processos fermentativos para obtenção de vários produtos, como ácido láctico, butanol e etanol, entre outros. Segundo Alegre (1988) o aproveitamento do soro para produção de etanol vem sendo estudado desde a década de 40, tendo início em 1941, com o cientista Browne.

Florentino et al. (2007) estudaram o uso do soro de queijo de coagulação enzimática como matéria-prima para produção de vinagre e os processos de fermentação alcoólica e fermentação acética. A primeira etapa consistiu no estudo da fermentação alcoólica testando-se os dois tipos de levedura: a Kluyveromyces lactis ATCC 5698 e a Saccharomyces cerevisiae, observando que a produção de etanol com a Saccharomyces, cultivada em soro acrescido de sacarose, foi aproximadamente quatro vezes maior do que a produção obtida com a Kluyveromyces lactis, passando a adotá-la com vista à produção de etanol.

Alguns problemas são encontrados quando se utiliza o soro como substrato para a fermentação alcoólica como, por exemplo, o número limitado de micro-organismos capazes de metabolizar diretamente a lactose com produção de etanol, bem como o fato desses micro-organismos serem inibidos por concentrações moderadas do substrato (glicose) e do produto (etanol) (Florentino, 2006).

Segundo Leite \& Leal (2007) o etanol já é, hoje, um grande sucesso no Brasil como substituto da gasolina e seu futuro é promissor com o advento de tecnologias de segunda geração. Pressionados pelos aumentos contínuos do preço do petróleo, benefícios que a expansão da utilização dos biocombustíveis pode trazer para o setor agrícola por meio da implantação de projetos específicos para fins energéticos com o objetivo de promover o desenvolvimento regional sustentável e redução das emissões de gás carbônico, diferentes países procuram participar no novo e potencial mercado de energias alternativas ao petróleo (Demirbas, 2007; Vertès et al., 2008, Masiero \& Lopes, 2008).

Tendo em vista o exposto objetivou-se, neste trabalho, a produção de etanol a partir do lactossoro industrial, do queijo coalho, utilizando a metodologia de planejamento fatorial e análises de superfície de resposta.

\section{Material e Métodos}

A matéria-prima utilizada no trabalho foi o soro resultante da produção de queijo "tipo Coalho" proveniente de uma Indústria de Laticínio localizada no Cariri Paraibano.

O soro foi coletado logo após sua obtenção, em recipientes plásticos e transportado para o NUPEA (Núcleo de Pesquisa e Extensão em Alimentos) do Departamento de Química do Centro de Ciências e Tecnologia da Universidade Estadual da Paraíba, onde foi desenvolvido este trabalho de pesquisa.

Inicialmente, o soro foi desproteinizado utilizando-se o método ácido e térmico, que consiste no abaixamento do $\mathrm{pH}$ do soro para 4,6, através do ácido cítrico e depois o aquecido a $90{ }^{\circ} \mathrm{C}$ (Florêncio et al., 2009a). Após obtenção do material floculado o soro foi mantido em repouso até atingir temperatura ambiente e o aglomerado proteico retirado por meio de filtração; em seguida, o soro foi analisado em termos de gordura, proteínas, lactose, sais minerais, extrato seco total e $\mathrm{pH}$; enfim, a metodologia utilizada é a descrita pelo LANARA (Brasil, 1981).

O micro-organismo utilizado foi a Saccharomyces cerevisiae (fermento comercial) da marca Fleischman ${ }^{\circledR}$, com $70 \%$ de umidade, obtido em supermercados da cidade de Campina Grande, PB.

A chaptalização é a etapa do processo em que se adiciona a sacarose ao mosto para aumentar a quantidade total de açúcar e assim elevar o potencial teor de álcool (Brasil, 1997). Sendo geralmente realizada quando o meio não tem quantidade suficiente de açúcares, como foi o caso do lactossoro, visto que a levedura em estudo não degrada a lactose. Neste estudo o soro serviu de substrato nutritivo para a levedura por ser uma fonte rica em carbono e nitrogênio, entre outros.

Avaliou-se quantitativamente a influência das variáveis de entrada (sacarose e levedura) sobre a resposta (\% de conversão) por meio de um planejamento fatorial $2^{2}$, totalizando 7 experimentos, sendo 4 distintos e 3 no ponto central, realizados em Erlenmeyer de $2.000 \mathrm{~mL}$, em triplicata. 
Com o planejamento fatorial simples observou-se que apenas a média dos resultados se apresentou estatisticamente significativa a nível de $95 \%$ de confiança utilizando-se o programa computacional Statistic 5.0. Como o modelo linear não foi estatisticamente significativo os níveis do planejamento foram ampliados acrescentando-se níveis axiais $(-\alpha,+\alpha)$ e se realizando um planejamento fatorial $2^{2}+$ configuração estrela (composto central) (Rodrigues \& Iemma, 2005). Com isto foi acrescentada a configuração estrela, ficando uma matriz de planejamento fatorial $2^{2}+3$ pontos centrais + configuração estrela, totalizando 11 experimentos. A Tabela 1 mostra a matriz com todos os experimentos.

Tabela 1. Matriz do planejamento fatorial $2^{2}$ com 3 repetições no ponto central mais configuração estrela (composto central)

\begin{tabular}{ccc}
\hline Experimento & Sacarose & Inóculo \\
1 & -1 & -1 \\
2 & +1 & -1 \\
3 & -1 & +1 \\
4 & +1 & +1 \\
5 & 0 & 0 \\
6 & 0 & 0 \\
7 & 0 & 0 \\
8 & $-1,4142$ & 0 \\
9 & $+1,4142$ & 0 \\
10 & 0 & $-1,4142$ \\
11 & 0 & $+1,4142$ \\
\hline
\end{tabular}

Os ensaios foram monitorados realizando-se as seguintes análises físico-químicas:

Teor alcoólico: Determinado por meio da leitura direta em ebuliômetro.

Açúcares redutores totais (ART): O método para obtenção de ART se baseia na redução do DNS (ácido 3,5 dinitrosalicílico) a ácido 3-amino-5-nitrosalicílico ao mesmo tempo com a oxidação do grupo aldeído do açúcar a grupo carboxílico. Com o aquecimento a solução se torna alaranjada ao ser lida por meio do equipamento espectrofotômetro a $540 \mathrm{~nm}$ (Miller, 1959).

Concentração celular: Na determinação da concentração celular adotou-se o método de massa seca, que foi determinada pela diferença entre o peso final e o peso inicial e expresso em $\mathrm{g} \mathrm{L}^{-1}$ (Tríboli, 1989). 2005).

pH: Determinado utilizando-se um pHmetro digital (Brasil,

Acidez total, acidez fixa e acidez volátil: As concentrações de acidez total e acidez fixa foram determinadas pelo método de titulação volumétrica (Brasil, 2005). A acidez volátil foi obtida pela diferença entre a acidez total e a acidez fixa.

Sólidos solúveis totais ( $\left.{ }^{\circ} \mathrm{Brix}\right)$ : A leitura foi realizada por meio de um refratômetro de Abbé (Brasil, 2005).

Percentual de conversão: $O$ percentual de conversão para produção de etanol a partir do lactossoro foi calculado por meio da Eq. 1.

$$
\text { \%conversão }=\frac{\left(\mathrm{P}_{\mathrm{f}}-\mathrm{P}_{\mathrm{i}}\right)}{\left(\mathrm{S}_{\mathrm{i}}-\mathrm{S}_{\mathrm{f}}\right) \times 0,511} \times 100
$$

em que:

$\mathrm{P}_{\mathrm{f}} \quad$ - concentração de etanol final
$\mathrm{P}_{\mathrm{i}} \quad$ - concentração de etanol inicial

$\mathrm{S}_{\mathrm{f}} \quad$ - concentração final do substrato

$\mathrm{Si}$ - concentração inicial do substrato

0,511 - fator estequiométrico. $1 \mathrm{~g}$ de ART produz $0,511 \mathrm{~g}$ de etanol.

\section{Resultados e Discussão}

A Tabela 2 apresenta os resultados da caracterização do soro de queijo após a remoção da proteína por meio do método ácido térmico.

Tabela 2. Médias dos componentes do soro de queijo desproteinizado

\begin{tabular}{lc}
\hline Componentes & Média \\
pH & $4,93 \pm 0,06$ \\
Gordura (\%) & Traços \\
Lactose (\%) & $3,57 \pm 0,06$ \\
Extrato seco total (\%) & $4,62 \pm 0,22$ \\
Sais minerais (\%) & $0,60 \pm 0,01$ \\
Proteína (\%) & $0,45 \pm 0,19$ \\
\hline
\end{tabular}

Para os resultados da Tabela 2 evidencia-se que ainda há uma alta concentração de lactose no soro (3,57\%). Analisando o pH encontrado, que foi de 4,93, ele está próximo ao observado por Florêncio et al. (2009b) ao trabalharem com adsorção e quantificação das proteínas do lactosoro por adição de quitosana e correlação percentual ao método de coagulação ácido térmico, que foi de $4,65 \%$, trabalhando com o mesmo método de separação das proteínas (ácido térmico), sendo o valor observado muito importante visto que a faixa de $\mathrm{pH}$ ótima para desenvolvimento da levedura se mantém na faixa de 4,0-4,5.

Observando os valores relatados para o teor de gordura (Tabela 2) constata-se que sua quantificação reduziu quase que totalmente o que representa um resultado satisfatório visto que a gordura não participa do processo fermentativo, constituindo-se um dos compostos que mais preocupam as indústrias de laticínios em virtude do seu alto poder poluidor, quando descartado sem tratamento prévio. Os valores encontrados são semelhantes aos achados por Florentino et al. (2007) que utilizaram a mesma temperatura e o mesmo método na precipitação, e por Florêncio et al. (2009b) comprovando, com isto, a eficiência na absorção do método ácido térmico.

A proteína encontrada no leite está em torno de 3,5 a 3,6\% (Behmer, 1991). O teor de $0,45 \%$ observado no soro de queijo "Tipo Coalho" desproteinizado, representa aproximadamente $12,5 \%$ das proteínas totais do leite que, muitas vezes, é desperdiçada.

$\mathrm{Na}$ Tabela 2 verifica-se que os teores encontrados para sais minerais $(0,60 \%)$ e extrato seco total $(4,62 \%)$, estão próximos aos indicados por Florentino et al. (2007) que trabalharam com soro de queijo "tipo Coalho" de queijeira artesanal evidenciando que, na produção de etanol, esses compostos servirão de nutrientes para o desenvolvimento do micro-organismo.

Os ensaios foram realizados em biorreatores acompanhando o processo por $48 \mathrm{~h}$, com adição de sacarose e inóculo em diferentes porcentagens, como mostra a Tabela 3, seguindo o planejamento fatorial $2^{2}+3$ pontos centrais + configuração 
estrela (composto central) e se estabelecendo, como variável resposta, a \% de conversão.

Tabela 3. Condições dos ensaios do planejamento fatorial $2^{2}+3$ pontos centrais + configuração estrela (composto central) com sua respectiva variável resposta

\begin{tabular}{cccc}
\hline Experimento & Sacarose & Inóculo & $\begin{array}{c}\text { \% de Conversão } \\
\text { (\%) }\end{array}$ \\
\cline { 2 - 3 } 1 & $-(100)$ & $-(3)$ & 70,77 \\
2 & $+(160)$ & $-(3)$ & 72,57 \\
3 & $-(100)$ & $+(9)$ & 52,80 \\
4 & $+(160)$ & $+(9)$ & 47,42 \\
5 & $0(130)$ & $0(6)$ & 54,10 \\
6 & $0(130)$ & $0(6)$ & 50,65 \\
7 & $0(130)$ & $0(6)$ & 47,80 \\
8 & $-1,4142(38)$ & $0(6)$ & 74,06 \\
9 & $+1,4142(222)$ & $0(6)$ & 55,01 \\
10 & $0(130)$ & $-1,4142(1,8)$ & 76,14 \\
11 & $0(130)$ & $1,4142(10,2)$ & 56,09 \\
\hline
\end{tabular}

Os valores da análise de variância para o modelo quadrático para 95\% de confiança estão apresentados na Tabela 4.

Tabela 4. Análise de variância para o modelo quadrático

\begin{tabular}{lrrrc}
\hline & SQ & GL & MQ & Teste F \\
Regressão & 1098,18 & 5 & 219,640 & 7,10 \\
Residuo & 154,71 & 5 & 30,942 & \\
Falta de ajuste & 0 & - & & \\
Erro puro & 154,71 & 5 & & \\
Total & 1252,93 & 10 & & \\
R $^{2}$ (\%) & 87,65 & & & 5,05 \\
F tabelado 0.95 .5 .5 & & & & \\
\hline
\end{tabular}

SQ - Soma quadrática; GL - Grau de liberdade; MQ - Média quadrática

O F calculado foi 7,10 e o F tabelado ${ }_{0.95 .5 .5}$ (Rodrigues \& Iemma, 2005) foi 5,05, observando que o modelo é estatisticamente significativo para $95 \%$ de confiança visto que a razão entre F calculado e F tabelado foi 1,40. Segundo Barros Neto et al. (2005) para que o modelo tenha validade estatística, de acordo com o Teste $F$, o valor da razão $F$ calculado em relação a F tabelado deve ser acima de 1,0. O modelo codificado está apresentado na Eq 2, com os coeficientes estatisticamente significativos em negrito. Observa-se, na Eq. 2, que o modelo apresenta os coeficientes com seus erros padrão em que $\mathrm{S}$ é a sacarose e I o inóculo.

$$
\begin{aligned}
\% \text { de Conversao } & =\mathbf{5 0 , 8 5} \pm \mathbf{3}, \mathbf{7 1}-3,82 \pm 1,97 \mathrm{~S}-\mathbf{8 , 9 3} \pm \mathbf{1}, \mathbf{9 7} \mathbf{L}- \\
& -1,80 \pm 2,78 \mathrm{SL}+5,73 \pm 2,34 \mathrm{~S}^{2}+\mathbf{6 , 5 2} \pm \mathbf{2 , 3 4 \mathbf { L } ^ { 2 }}
\end{aligned}
$$

A Figura 1 apresenta o gráfico de Pareto com nível de $95 \%$ de confiança para a estimativa dos efeitos.

Na Figura 1 verifica-se que o efeito da concentração de inóculo, tanto para o efeito principal como para o quadrático, mostrou-se estatisticamente significativo. Com relação aos outros efeitos (sacarose quadrática, sacarose linear e interação entre as variáveis sacarose e inóculo) não apresentaram significância estatística a nível de $95 \%$ de confiança.

A Figura 2 apresenta a superfície de resposta gerada pelo programa Statistic 5.0 com base na variável dependente do percentual de conversão.

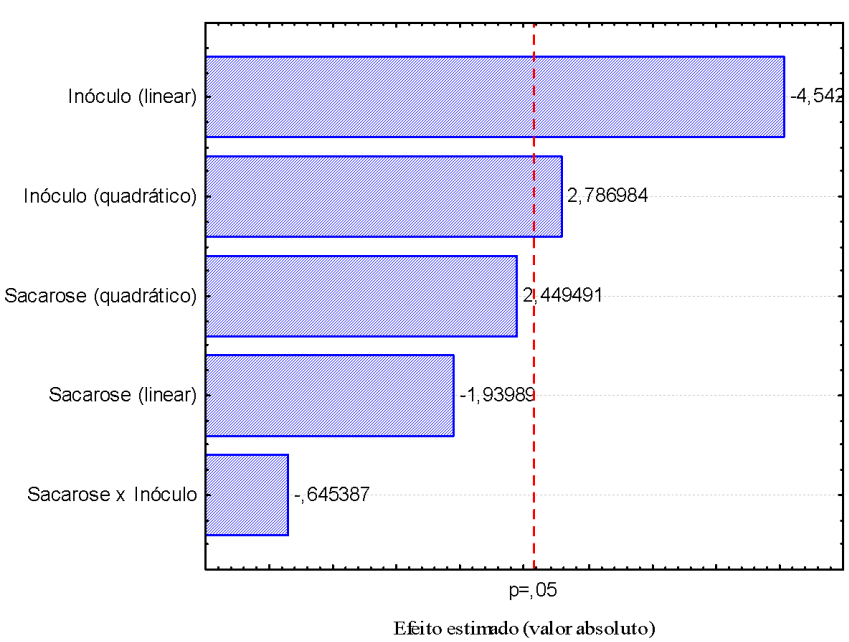

Figura 1. Gráfico de Pareto para o \% de conversão

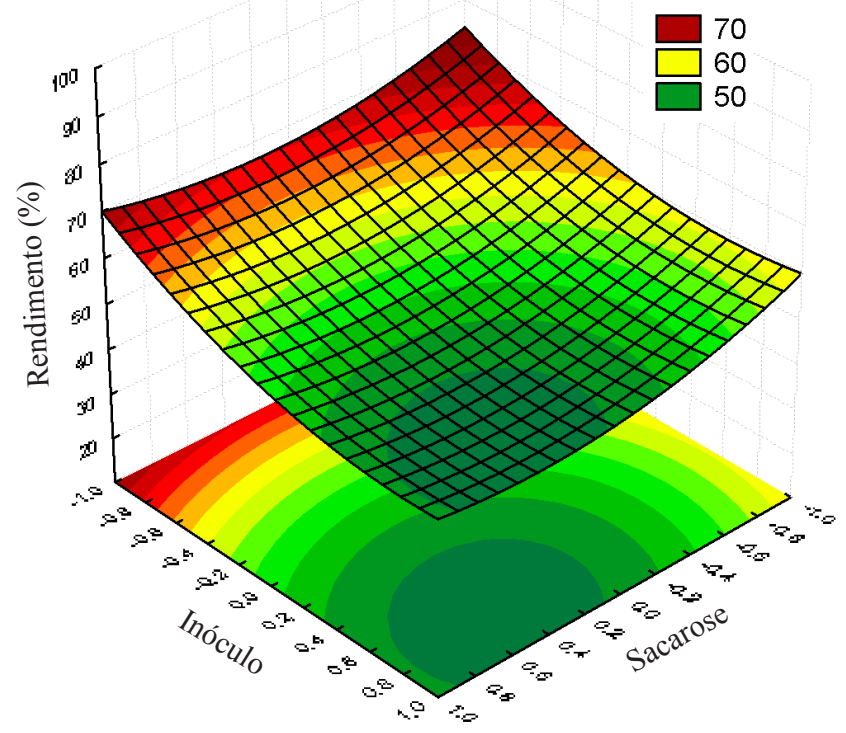

Figura 2. Superfície de resposta para o \% de conversão

Observa-se, na Figura 2 que, fixando o valor da concentração de inóculo abaixo de $6 \mathrm{~g} \mathrm{~L}^{-1}$ e concentração inicial de sacarose em $100 \mathrm{~g} \mathrm{~L}^{-1}$, o \% de conversão é máximo para o processo em estudo (76,14\%). Florentino et al. (2007) obtiveram, ao trabalhar com o mesmo inóculo na concentração de $100 \mathrm{~g} \mathrm{~L}^{-1} \mathrm{e}$ adicionando $100 \mathrm{~g} \mathrm{~L}^{-1}$ de sacarose, um percentual de conversão máximo de $14,02 \%$ sinalizando que o processo do presente estudo se mostrou mais eficiente.

Lopes \& Silva (2006) obtiveram, trabalhando com a elaboração de fermentados a partir do figo-da-india, um percentual de conversão máximo de $100 \%$ para a polpa do fruto da palma forrageira, com adição de $15 \mathrm{~g} \mathrm{~L}^{-1}$ de levedura (Saccharomyces cerevisiae) e $11^{\circ}$ Brix, percentual este bem superior ao encontrado neste estudo.

A adição de sacarose é necessária haja vista que a levedura Saccharomyces cerevisiae não utiliza a lactose presente no meio mas açúcares fermentescíveis (glicose e frutose), que são produtos da hidrólise da sacarose no processo de fermentação alcoólico (Vertès et al., 2008; Gabardo et al., 2011). O meio 
soro de queijo desproteinizado mostrou-se adequado para a produção de etanol.

\section{CONCLUSÕES}

1. O método ácido térmico apresentou-se satisfatório para a remoção das proteínas do lactossoro do queijo tipo coalho.

2. O melhor percentual de conversão $(76,14 \%)$ do soro do queijo tipo coalho foi obtido com a adição de $100 \mathrm{~g} \mathrm{~L}^{-1}$ de sacarose e $6,0 \mathrm{~g} \mathrm{~L}^{-1}$ de levedura evidenciando, assim, uma alternativa tecnicamente viável para produção de etanol no País, além de reduzir o potencial poluidor deste resíduo.

\section{AgradeCIMENTOS}

Ao Conselho Nacional de Desenvolvimento Científico e Tecnológico $(\mathrm{CNPq})$ pelo apoio financeiro a esta pesquisa.

\section{Literatura Citada}

Abreu, L. R. Leite e derivados - Caracterização físico-química, qualidade e legislação. Textos acadêmicos. Lavras: UFL/ FAEPE, 2005. 151p.

Alegre, R. M. Contribuição ao estudo de aproveitamento de soro de queijo para produção de lactose e etanol. Campinas: UNICAMP, 1988. 178p. Dissertação Mestrado

Barros Neto, B. de; Scarmínio, I. S.; Bruns, R. E. Planejamento e otimização de experimentos. 2 ed. Campinas: UNICAMP, 2005, 299p.

Behmer, M. L. A. Tecnologia do leite. 15.ed., São Paulo: Nobel, 1991, 320p.

Brasil. Ministério da Agricultura, Pecuária e Abastecimento. Secretaria Nacional de Defesa da Agropecuária. Laboratório Nacional de Referência Animal - LANARA. Portaria n. 1 de 7/10/1981. Métodos analíticos oficiais para controle de produtos de origem animal e ingredientes: Métodos físicoquímicos. Brasília: Congresso Nacional, 1981.

Brasil, Ministério da Agricultura, Pecuária e Abastecimento. Decreto n.2.314 de 04 de setembro de 1997. Brasília: Congresso Nacional, 1997. 1p.

Cavalcante, J. F. M.; Andrade, N. J. de.; Furtado, C. L. de L. F.; Ferreira, C. L. de O.; Pinto, E. E. Processamento do queijo coalho regional empregando leite pasteurizado e cultura lática endógena. Ciência e Tecnologia de Alimentos, v.27, p.205-214, 2007.

Demirbas, A. Progress and recent trends in biofuels. Progress in Energy and Combustion Science, v.33, p.1-18, 2007.

Florêncio, I. M.; Martins, R. S.; Araújo, A. dos S.; Cavalcanti, M. T.; Silva, F. L. H. da; Florentino, E. R. produzido no semi-árido do Rio Grande do Norte, no cariri e no agreste paraibano para aplicabilidade industrial. Higiene Alimentar, v.23, p.138-139, 2009a.

Florêncio, I. M.; Martins, R. S. ; Silva, R. A. dos S. ; Araújo, M. de S. ; Alves, R. M.; Florentino, E. R. Adsorção e quantificação das proteínas do lactosoro por adição de quitosana e correlação percentual ao método de coagulação ácido-térmico. Química no Brasil, v.3, p.1-15, 2009b.

Florentino, E. R. Aproveitamento do soro de queijo de coagulação enzimática. Natal: UFRN, 150p. 2006. Tese Doutorado
Florentino, E. R.; Batista, J. A.; Santos, E. S.; Macedo, G. R. Utilização do soro de queijo na produção de etanol utilizando Klyveromyces lactis. Higiene Alimentar, v.21, p.36-37, 2007.

Florentino, E. R.; Macedo, G. R. de; Santos, E. S. dos. Caracterização do soro de queijo visando processo de aproveitamento. Higiene Alimentar, v.19, p.30-32, 2005.

Gabardo, S.; Rech, R.; Ayub, M. A. Z. Determination of lactose and ethanol diffusion coefficients in calcium alginate gel spheres: Predicting values to be used in immobilized bioreactors. Journal of Chemical and Engineering Data, v.56, p.2305-2309, 2011.

Leite, R. C.; Leal, M. R. L. O biocombustível no Brasil. Novos estudos: CEBRAP, n.78, p.15-21, 2007.

Lopes, R. de V. V.; Silva, F. L. H. da. Elaboração de fermentados a partir do figo-da-índia. Revista de Bilogia de Ciência da Terra, v.6, p.305-315, 2006.

Madrona, G. S.; Terra, C. O.; Penha, C. B. Efeito da substituição do açúcar por oligofrutose em bebida láctea achocolatada. Revista Brasileira de Tecnologia Agroindustrial, v.3, p.2937, 2009.

Masiero, G.; Lopes, H. Etanol e biodiesel como recursos energéticos alternativos: perspectivas da América Latina e da Ásia. Revista Brasileira de Política Internacional, v.51, p.60-79, 2008.

Miller, G. L. Utilização de reagente para a determinação de ácido dinitrossalicílico de açúcares redutores, Analitycal Chemistry, v.31, p.426-428, 1959

Richards, N. S. P. Soro lácteo: Perspectivas industriais e proteção ao meio ambiente. Revista Food Ingredientes, n.17, p.20-24, 2002.

Rodrigues, M. I.; Iemma, A. F. Planejamento de experimentos e otimização de processos: Uma estratégia seqüencial de planejamentos. 1 ed. Campinas: Editora Casa do Pão, 2005. 326p.

Santos, J. P. V.; Ferreira, C. L. L. F. Alternativas para o aproveitamento de soro de queijo nos pequenos e médios laticínios. Revista do Instituto de Lacticínios, v.56, p.44$50,2001$.

Silva, C. A.; Herman-Gomez, R. C. Qualidade protéica do soro de leite fermentado pela levedura Kluyveromyces fragilis. Ciência Rural, v.30, p.515-520, 2000.

Triboli, E. P. D. R. Métodos analíticos para o acompanhamento da fermentação alcoólica. São Caetano do Sul: Laboratório de Engenharia Bioquímica e de Alimentos, Escola de Engenharia de Mauá, Instituto Mauá de Tecnologia. 1989. 52p.

Valduga, E.; Paviani, L. C.; Mazur, S. P.; Finzer, J. R. D. Aplicação do soro de leite em pó na panificação. Alimentos e Nutrição, v.17, p.393-400, 2006.

Vertès, A. A.; Massayuki, I.; Yukawa, H. Technological options for biological fuel ethanol. Journal of Molecular Microbiology and Biotechnology, v.15, p.16-30, 2008. 